\title{
The effects of surface anaesthesia on the autonomic dysreflexia response during functional electrical stimulation
}

\author{
JM Matthews, GD Wheeler, RS Burnham, LA Malone and RD Steadward \\ Rick Hansen Centre, W1-67 Van Vliet Centre, University of Alberta, Edmonton, Alberta, Canada T6G 2 H9
}

Recently, increases in blood pressure (BP) and concomitant bradycardia, suggestive of autonomic dysreflexia (AD), have been documented during functional electrical stimulation (FES) in individuals with a high spinal cord injury (SCI). If uncontrolled, this response could preclude the safe use of FES among such individuals. FES induced pain is partly related to stimulation of skin nociceptors. Therefore, measures to reduce skin sensitivity may reduce the risk of AD during FES. The purpose of this study was to determine if topical anaesthetic applied over the site of electrical stimulation could minimize the AD cardiovascular and hormonal responses to FES in individuals with SCI above the T6 level.

Seven subjects with a SCI above T6 received FES to the quadriceps muscle of each leg under two conditions on two different testing days. The two treatment conditions, topical anaesthetic and placebo creams, were double blinded and randomized. The cream was administered to an area the size of the electrode $(10 \times 10 \mathrm{~cm}) 1 \mathrm{~h}$ prior to stimulation. Stimulation began at $0 \mathrm{mAmps}$ and increased by $16 \mathrm{mAmps}$ every 2 min until an intensity of $160 \mathrm{mAmps}$ was achieved. HR and BP were measured at each stimulation intensity level. Catecholamines were analyzed three times during the stimulation protocol (pre, mid and post stimulation intensities). At the end of the stimulation protocol, FES induced isometric quadriceps contraction force at $160 \mathrm{mAmps}$ intensity was measured using a hand held dynamometer.

As FES stimulation intensity increased, significant rises in systolic and diastolic BP were seen, with a concomitant progressive drop in HR. The AD response to stimulation was not significantly different between the topical anaesthetic and placebo conditions. Serum catecholamine (epinephrine and norepinephrine) levels tended to rise with increasing FES intensity levels but did not reach statistical significance. The two treatment conditions did not significantly affect serum catecholamine levels or FES-induced quadriceps contraction force.

In summary, FES application to the quadriceps muscle in high level SCI subjects resulted in significant increases in BP, decreases in HR (AD-like response), a trend towards elevations in catecholamine levels, and no difference in quadriceps muscular strength. However, these responses were unaffected by the use of topical anaesthetic cream on the skin at the stimulation site. This suggests that other mechanisms than skin nociception are operative in FES-induced AD.

Keywords: functional electrical stimulation; spinal cord injury; autonomic dysreflexia; anaesthetic; catecholamine; heart rate; blood pressure

\section{Introduction}

In the early $1970 \mathrm{~s}$, the use of electrical current to stimulate paralysed musculature, a technique known as Functional Electrical Neuromuscular Stimulation (FES), was identified as a treatment modality that could have significant benefits for spinal cord injured (SCI) patients. ${ }^{1,2}$ Since then, FES has proven to be an effective means of eliciting functional movement in paralysed limbs and has the potential to reverse or alleviate many of the health problems associated with

Correspondence: Dr Garry Wheeler, Ph.D. paralysis due to SCI. ${ }^{3}$ Uses of FES in individuals with SCI may generally be categorized into three areas: rehabilitation, prevention and exercise (for a recent comprehensive review, see ${ }^{4}$ ).

To date, most reports regarding the use of FES in rehabilitation, prevention and exercise settings have been positive. However, recent evidence suggests that FES assisted training or therapeutic interventions may induce autonomic dysreflexia (AD) in high level SCI patients. ${ }^{5}$ AD occurs when a nociceptive stimulus distal to the level of the SCI triggers a mass sympathetic discharge, resulting in hypertension, piloerection, sweating and anxiety. This response has 
been shown to be largely norepinephrine mediated. ${ }^{6} \mathrm{~A}$ reactive parasympathetic component manifest by bradycardia, facial vasodilatation and vascular headache is also characteristic of the syndrome. Uncontrolled AD can result in serious health complications such as seizures, blindness or death, ${ }^{7}$ which would pose an unacceptable risk to FES. Prior to Ashley et $a l^{5}$ reporting the association between FES and AD, the risk for FES-associated $\mathrm{AD}^{8}$ and exercise-induced hypertension ${ }^{9}$ were dismissed as infrequent and relatively unimportant. In contrast, it has been the experience in our laboratory that, when monitoring heart rate (HR) and blood pressure (BP) continuously during FES with SCI patients, an AD response to FES is commonplace. Any method to reduce the magnitude of the blood pressure change with FES, or the intensity of the FES nociception perceived in sensory incomplete SCI patients, would make FES training safer and more widely applicable.

During FES, electrical current is passed from the surface electrodes through the skin into the muscle. This current causes the underlying motor nerves to discharge, thereby causing the muscles to contract. Theoretically, FES nociception could come from stimulation of one or a combination of the following: electrical activation of pain fibres (A delta and C fibres) of the skin, or muscle; isometric muscle contraction and its resultant ischemia, metabolites and/or musculotendinous stress. Currently, researchers examining the use of electrical muscle stimulation in able-bodied populations have attempted to produce a relatively low pain, yet effective contraction-inducing electrical stimulus. ${ }^{10,11}$ Another approach would be to reduce the pain sensitivity of the skin. The use of topical skin anaesthetic cream prior to invasive skin procedures such as venipuncture, arterial puncture or skin surgical procedures, has recently gained popularity. ${ }^{12} \mathrm{~A}$ published report described one such cream (EMLA, a mixture of lignocaine and prilocaine - Astra Pharma Inc.) being applied to the skin of quadriplegics' legs in an attempt to reduce 'reflex muscle spasm induced by the stimulus' thereby allowing more accurate FES-induced measurements of muscle contractile properties. ${ }^{13}$ If this type of local anaesthetic cream could effectively reduce the FES induced skin nociception, suppression of the AD response may result. However, the overall advantage of this type of local anaesthetic cream would be diminished if the strength of the FES induced muscle contraction was reduced.

The purpose of this study therefore, was to determine if local anaesthetic cream at the site of FES application would: (a) reduce the AD-related cardiovascular response; (b) reduce the magnitude of the sympathetic output; and (c) affect the strength of the FES-induced quadriceps contraction.

\section{Methodology}

Prior to participation, all subjects were screened for contraindications to lower extremity FES, were informed of the nature and protocol of the study, and signed an informed consent. Seven subjects (six males, one female) with complete motor and sensory tetraplegia participated. Subject characteristics are summarized in Table 1. Each testing session lasted approximately $2.5 \mathrm{~h}$. Subjects arrived at the testing area $30 \mathrm{~min}$ prior to testing, having avoided any strenuous activity, caffeine intake for at least $12 \mathrm{~h}$, and having recently emptied their bladder. Prior to testing each subject was familiarized with the testing equipment and procedures, and the Rick Hansen Centre emergency medical protocol was followed in the event of uncontrolled AD. ${ }^{14}$

\section{Experimental design}

A two factor (cream type $\times$ stimulus intensity) repeated measures design was utilized. Subjects were tested on two separate sessions - once using anaesthetic (EMLA) cream, and once using placebo cream. At each session, each leg was tested separately and was considered a quasi-independent limb in the analysis. The application of EMLA cream or placebo was double-blinded, and the order of application was randomly determined for each subject by the pharmacist, and the codes for the creams were broken at the conclusion of the investigation.

Table 1 Subject profile

\begin{tabular}{|c|c|c|c|c|c|c|c|}
\hline ID \# & $\begin{array}{l}\text { Level } \\
\text { of injury }\end{array}$ & $\begin{array}{l}\text { Time post } \\
\text { injury (yrs) }\end{array}$ & Age (yrs) & $\begin{array}{l}\text { Prior use } \\
\text { of FES }\end{array}$ & $\begin{array}{l}\text { Episodes } \\
\text { of } A D\end{array}$ & $\begin{array}{l}\text { Signs or } \\
\text { symptoms } \\
\text { of AD }\end{array}$ & $\begin{array}{l}\text { Sources } \\
\text { of } A D\end{array}$ \\
\hline 1 & $\mathrm{C} 7 / \mathrm{T} 1$ & 4.5 & 31 & $\mathrm{Y}$ & Y & FES, full bladder & chills \\
\hline 2 & $\mathrm{C} 6 / 7$ & 21 & 38 & $\mathrm{Y}$ & $\mathrm{N}$ & & \\
\hline 3 & $\mathrm{C} 6 / 7$ & 5 & 34 & $\mathrm{~N}$ & $\mathrm{~N}$ & & \\
\hline 4 & $\mathrm{C} 4 / 5$ & 4.5 & 44 & $\mathrm{Y}$ & $\mathrm{Y}$ & FES, exercise & $\begin{array}{l}\text { elevated blood pressure, } \\
\text { pounding headache }\end{array}$ \\
\hline 5 & $\mathrm{C} 6 / 7$ & 3 & 31 & $\mathrm{~N}$ & Y & bladder infection & headache, seizure \\
\hline 6 & C6 & 7.5 & 23 & $\mathrm{~N}$ & Y & bladder & headache \\
\hline 7 & $\mathrm{C} 5 / 6$ & 15 & 38 & $\mathrm{~N}$ & $\mathrm{Y}$ & $\begin{array}{l}\text { pressure, bowel and } \\
\text { bladder }\end{array}$ & $\begin{array}{l}\text { headache, sweating, goose- } \\
\text { flesh, racing heart }\end{array}$ \\
\hline
\end{tabular}


The anaesthetic and placebo were in identical cream form. Five grams of cream were applied under occlusion to the skin overlying the quadriceps muscle. The cream covered an area of the skin corresponding to the size of the FES electrode, and provided analgesia to a depth of $3 \mathrm{~mm} .{ }^{12}$ After $1 \mathrm{~h}$ of application time, the occlusive dressing was removed, the topical cream wiped off, and the area cleansed with an alcohol swab in preparation for FES. For consistency between the test days, the location of the cream application and electrode placement was standardized by measurement of a set distance from the proximal pole of the patella for each subject. The muscle stimulator consisted of a four-channel neuromuscular stimulation device (Quadstim, Biomech Industries Edmonton Ltd.), which was connected to the subject via commercially available, reusable $\left(1.5^{\prime \prime} \times 3.5^{\prime \prime}\right)$ surface electrodes (Chattanooga Corp., $\mathrm{TN})$. The proximal electrode was positioned over the motor point and the distal electrode over the musculotendinous junction of the quadriceps muscle to elicit knee extension. Stimulation parameters included square monophasic waves of $250 \mathrm{msec}$ pulse width, $30 \mathrm{~Hz}$ frequency, 0 to $160 \mathrm{mAmps}$ stimulus intensity, at a $50 \%$ duty cycle (set at $4 \mathrm{~s}$ on/off time). Subjects were comfortably seated with $90^{\circ}$ of hip and knee flexion, and the feet were free from touching the floor. The graded FES protocol began at $0 \mathrm{mAmps}$ and increased by intervals of $16 \mathrm{mAmps}$ with a stimulation on-time of one minute followed by $3 \mathrm{~min}$ of off-time (rest), the minimum work: rest ratio to allow for recovery.

The subject's HR and BP were measured. BP was recorded manually by auscultation for all subjects by the research physician, and $\mathrm{HR}$ was measured with a PE sport tester (Polar Pacer HR Monitor, Model 45930, Polar Electro, Finland). BP and HR were measured at baseline, and thereafter at each intensity level during stimulation on-time.

In addition to recording $\mathrm{HR}$ and $\mathrm{BP}$, blood for measurement of serum catecholamines (epinephrine and norepinephrine) was drawn by the physician through a 24 gauge indwelling heparin-lock intravenous catheter placed into one of the subject's hands. A $10 \mathrm{ml}$ blood sample was taken for catecholamine levels on three occasions during the stimulation protocol: baseline - after sitting quietly for $30 \mathrm{~min}$ (0 mAmps), during stimulation on-time at $50 \%$ stimulation intensity (80 mAmps), and during stimulation ontime at $100 \%$ stimulation intensity (160 mAmps). The sample was drawn during the work phase of the work : rest cycle. Each blood sample was aliquoted for analysis. The tubes of blood were centrifuged to separate the serum, and the sample for haematocrit was taken immediately in an heparinized micro pipette and stored at $-20^{\circ} \mathrm{C}$. The sample was treated for catecholamine assay using High-Pressure Liquid Chromatography Separation with Electrochemical Detection. ${ }^{15}$ At the end of the stimulation protocol, the subject rested for $15 \mathrm{~min}$ and a recording of FESinduced maximal isometric knee extension force at maximal stimulation was made by the same experienced tester using a hand held dynamometer (Nicholas MMT, Lafayette, IN), as per the protocol of Bohannon. $^{18}$

On the subject's second visit, the above protocol was repeated under the second cream condition. The testing sessions were conducted at the same time each day.

\section{Statistical analysis}

A two factor (cream type $\times$ stimulus intensity) analysis of variance, with repeated measures on the second

Table 2 Effect of local anaesthetic cream on blood pressure, heart rate and cartecholamine responses to FES: Analysis of Variance

\begin{tabular}{|c|c|c|c|c|c|c|c|}
\hline Measure & Effect & $d f$ Effect & $\begin{array}{c}\text { Mean } \\
\text { square effect }\end{array}$ & $d f$ Error & $\begin{array}{c}\text { Mean } \\
\text { square effect }\end{array}$ & $F$ ratio & $P$ level \\
\hline \multirow[t]{3}{*}{ Heart rate } & Type of Cream & 1 & 468.8 & 26 & 905.1 & 0.52 & 0.48 \\
\hline & Graded Stimulus Intensity & 10 & 305.1 & 260 & 38.2 & 7.99 & 0.0000 \\
\hline & Interaction & 10 & 47.2 & 260 & 38.2 & 1.24 & 0.27 \\
\hline \multirow{3}{*}{$\begin{array}{l}\text { Systolic blood } \\
\text { pressure }\end{array}$} & Type of Cream & 1 & 263.7 & 26 & 5482.7 & 0.05 & 0.83 \\
\hline & Graded Stimulus Intensity & 10 & 3758.7 & 260 & 218.0 & 17.24 & 0.0000 \\
\hline & Interaction & 10 & 137.4 & 260 & 218.0 & 0.63 & 0.79 \\
\hline \multirow{3}{*}{$\begin{array}{l}\text { Diastolic blood } \\
\text { pressure }\end{array}$} & Type of Cream & 1 & 459.0 & 26 & 2482.8 & 0.18 & 0.67 \\
\hline & Graded Stimulus Intensity & 10 & 1464.3 & 260 & 87.6 & 16.72 & 0.0000 \\
\hline & Interaction & 10 & 37.0 & 260 & 87.6 & 0.42 & 0.94 \\
\hline \multirow[t]{3}{*}{ Norepinephrine } & Type of Cream & 1 & 0.1 & 26 & 3.8 & 0.02 & 0.89 \\
\hline & Graded Stimulus Intensity & 2 & 1.4 & 52 & 0.6 & 2.21 & 0.12 \\
\hline & Interaction & 2 & 0.4 & 52 & 0.6 & 0.60 & 0.55 \\
\hline \multirow[t]{3}{*}{ Epinephrine } & Type of Cream & 1 & 9.3 & 26 & 4084.9 & 0.00 & 0.96 \\
\hline & Graded Stimulus Intensity & 2 & 3889.6 & 52 & 1462.8 & 2.66 & 0.08 \\
\hline & Interaction & 2 & 383.6 & 52 & 1462.8 & 0.26 & 0.77 \\
\hline
\end{tabular}

Statistical significance at $P<0.05$ 
factor (intensity) was used to test for significant differences in BP (systolic and diastolic), HR and catecholamine responses (Table 2). A dependent t-test was used to analyze the difference in FES-induced knee extension force under the two cream conditions. Statistical significance was set at an alpha level of 0.05 .

\section{Results}

As stimulation intensity levels increased from 0 to $160 \mathrm{mAmps}$ for all SCI subjects, statistically significant decreases in HR and increases in systolic and diastolic BP, typical of the previously reported FES-induced AD response, were seen (Figures 1, 2 and 3). Similarly, there was a trend toward increases in catecholamines (epinephrine and norepinephrine) as stimulus intensity increased, although statistical significance was not achieved (Table 3). However, no difference in the $\mathrm{HR}, \mathrm{BP}$ or catecholamine responses was seen between the two cream conditions. Likewise, the two cream conditions did not significantly affect the FES-induced knee extension force output between anaesthetic cream (21.8 $\mathrm{kg} \pm 12.4)$, and placebo cream (21.2 kg士11.8).

\section{Discussion}

The purpose of this investigation was to evaluate the effect of topical local anaesthetic cream on FESinduced cardiovascular, hormonal and strength responses. We found that FES applied to the quadriceps muscle in quadriplegics results in a significant increase in $\mathrm{BP}$ and decrease in $\mathrm{HR}$, as previously described by Ashley et $a l^{5}$ We also detected a trend toward increases in both epinephrine and norepinephrine levels. This again confirms that FES can induce an AD-like response in individuals with high SCI. It is important to monitor for extreme BP changes associated with FES-induced AD. However, it does not appear that premedicating the site of FES application with topical local anaesthetic is a successful strategy to reduce the AD response in subjects such as participated in our study.

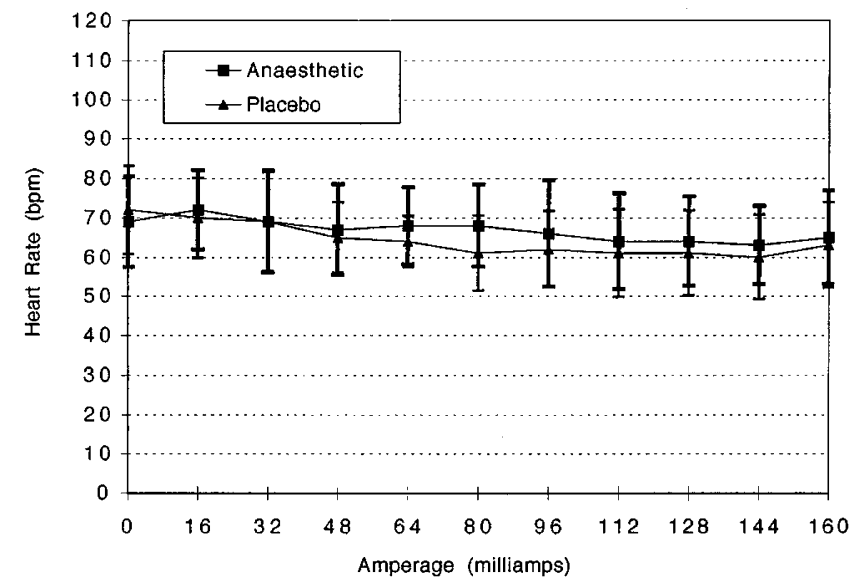

Figure 1 Heart rate response during FES
The SCI subjects in this study were recruited on the basis of availability and lesion level being above T6. None of them demonstrated dangerous hypertensive

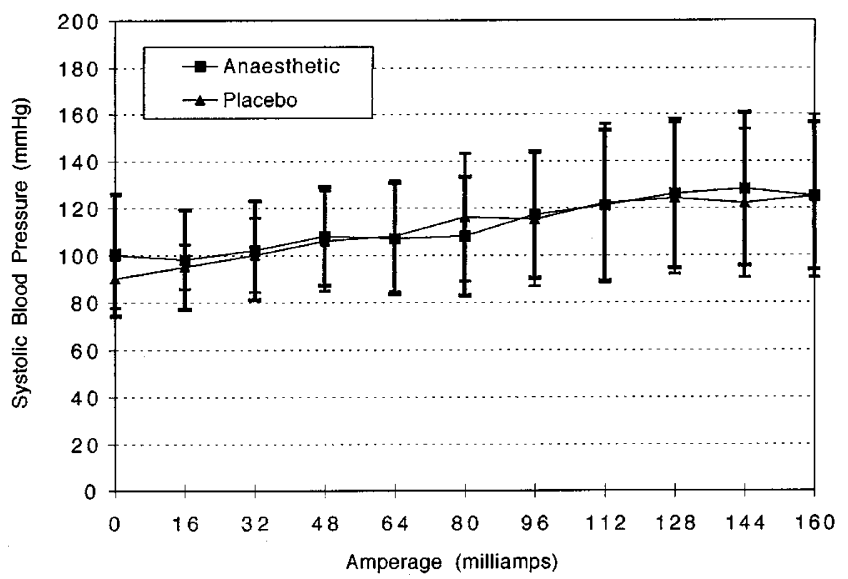

Figure 2 Systolic blood pressure response during FES

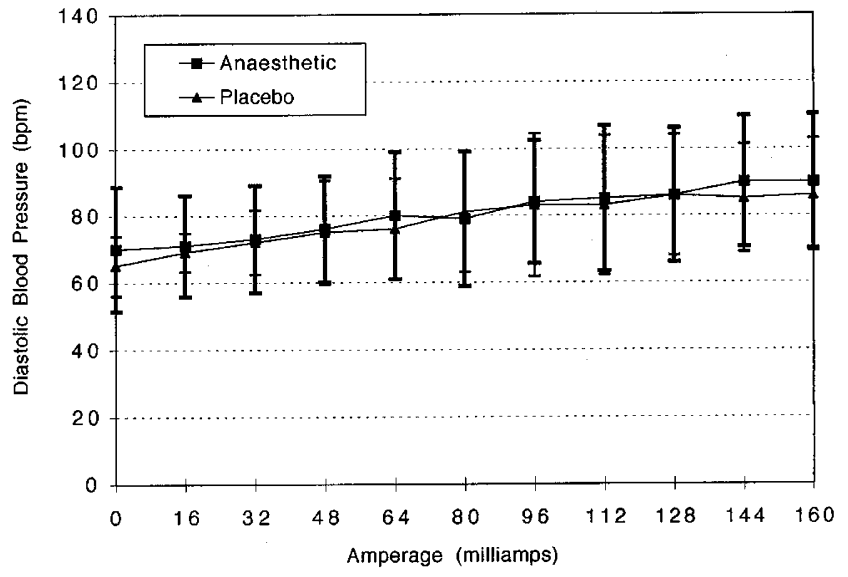

Figure 3 Diastolic blood pressure response during FES

Table 3 Catcholamine analysis

\begin{tabular}{|c|c|c|c|c|}
\hline \multicolumn{2}{|c|}{ Catecholamine } & $\begin{array}{l}\text { Pre } 0 \\
\text { mAmps }\end{array}$ & $\begin{array}{l}\text { Mid } 80 \\
\text { mAmps }\end{array}$ & $\begin{array}{l}\text { Post } 160 \\
\text { mAmps }\end{array}$ \\
\hline \multirow{6}{*}{$\begin{array}{l}\text { Epinephrine } \\
\text { pmol/L }\end{array}$} & EMLA & & & \\
\hline & mean & 94.29 & 101.64 & 120.57 \\
\hline & SD & 30.84 & 36.40 & 59.86 \\
\hline & Placebo & & & \\
\hline & mean & 92.36 & 108.93 & 113.21 \\
\hline & $\mathrm{SD}$ & 41.87 & 61.27 & 51.40 \\
\hline \multirow{5}{*}{$\begin{array}{l}\text { Norepinephrine } \\
\mathrm{nmol} / \mathrm{L}\end{array}$} & EMLA & & & \\
\hline & mean & 1.66 & 1.43 & 1.88 \\
\hline & SD & 1.71 & 1.11 & 1.22 \\
\hline & Placebo & & 156 & 188 \\
\hline & SD & $\begin{array}{l}1.53 \\
1.29\end{array}$ & $\begin{array}{l}1.50 \\
1.23\end{array}$ & $\begin{array}{l}1.00 \\
1.17\end{array}$ \\
\hline
\end{tabular}

*Statistical significance at $P<0.05$ 
responses to FES, in contrast to some of the subjects studied by Ashley et al, ${ }^{5}$ who responded with BP as high as $220 / 100 \mathrm{mmHg}$ and decreases in $\mathrm{HR}$ as much as $40 \%$. The seven SCI subjects who participated in this study could be classified as 'mild' AD responders to FES. This study did not determine whether local anaesthetic cream would be helpful for individuals with SCI who demonstrate severe AD reactions to FES, or who have trouble tolerating FES because of partial sensory sparing. Another explanation for the limited BP elevations, bradycardia and catecholamine response seen in the subjects of this study was that they received less total nociceptive stimulus. We measured the response to FES applied to a single leg whereas prior studies described greater FES-induced AD responses as a result of bilateral leg stimulation. ${ }^{5}$ The power of this study is limited by the reduced effect of the FES protocol used and the small number of subjects.

The lack of efficacy of the topical anaesthetic cream in suppressing the AD response to FES may be because the nociceptive trigger for FES-induced AD largely comes from the pain of the muscle and not skin stimulation. Topical anaesthetic has been reported to reduce pain up to a depth of $3 \mathrm{~mm}$ by reducing free nerve ending sensitivity. This was supported by findings of a pilot project conducted by Matthews et $a l,{ }^{17}$ in which FES was administered to neurologically normal subjects under topical anaesthetic and placebo conditions, using a similar protocol as described in this study. At lower stimulation intensities, the topical anaesthetic was effective in reducing the subjects' perceived pain, HR and BP response, but the topical anaesthetic lost its efficacy at the higher levels of FES intensity. Thus, FES-induced AD changes in SCI subjects are probably related to more complex mechanisms than skin nociception. High intensity FES results in a tetanic muscle contraction which, in addition to skin nociception, results in contractile unit pain on the basis of muscle ischemia, metabolite accumulation and/or musculotendinous strain. Similar findings have been suggested by Delitto et al. ${ }^{10}$

It is interesting to note that FES-induced elevations in BP were modest and served to raise the SCI subjects' BP from the typical hypotensive level to a normotensive level for someone who is neurologically intact. This raises the interesting possibility of using FES to treat SCI-related hypotension - a concept that warrants further study.

\section{Conclusion}

This study has demonstrated that FES application to the quadriceps muscle in high level SCI subjects results in significant increases in BP, decreases in $\mathrm{HR}$ and trends toward elevations in catecholamine levels (ADlike response). However, this $\mathrm{AD}$-like response is unaffected by the use of topical anaesthetic cream on the skin at the stimulation site. This suggests that other mechanisms than skin nociception are operative in FES-induced AD.

\section{Acknowledgements}

The authors wish to acknowledge and thank the following for their contributions in support of this research project: Astra Pharmaceuticals Incorporated, Mississauga, Ontario; Spinal Cord Injury Treatment Centre Society (SCITCS), Edmonton, Alberta; Department of Laboratory Medicine, Foothills Hospital, Calgary, Alberta; Lisa Olenik, Lara Bloxham, Helen Szaszkiewicz for data collection, and the subjects who volunteered to participate.

\section{References}

1 Petrofsky JS, Brown SW, Cerrel-Bazo H. Active physical therapy and its benefits in rehabilitation. Palaestra 1992; 8: $23-27$ and $61-62$.

2 Petrofsky JS, Phillips CA. The use of functional electrical stimulation for rehabilitation of spinal cord injured patients. Central Nervous System Trauma 1984; 1: $57-73$.

3 Cybulski GR, Penn RD, Jaeger RJ. Lower extremity functional neuromuscular stimulation in cases of spinal cord injury. Neurosurgery 1984; 15: $132-146$.

4 Andrews BJ, Wheeler GW. Functional and therapeutic benefits of electrical stimulation and spinal injury. Current Opinion in Neurology 1995; 8: $461-466$.

5 Ashley EA et al. Evidence of autonomic dysreflexia during functional electrical stimulation in individuals with spinal cord injuries. Paraplegia 1993; 31: 593-605.

6 Burnham R et al. Intentional induction of autonomic dysreflexia among quadriplegic athletes for performance enhancement: efficacy, safety, and mechanism of action. Clinical Journal of Sport Medicine 1994; 4: 1-10.

7 Ericksson RP. Autonomic Hyperreflexia: Pathophysiology and medical management. Archives of Physical Medicine and Rehabilitation 1980; 61: $431-440$.

8 Burke DC, Murray DD. Proceedings: The management of thoracic and thoraco-lumbar injuries of spine with neurological involvement. Journal of Bone \& Joint Surgery - British Volume 1975; 57: 254

19 Ragnarsson KT. Physiologic effects of FES-induced exercises in spinal cord injured individuals. Clinical Orthopaedics 1988; 233: $53-63$.

10 Delitto A, Strube MJ, Shulman AD, Minor SD. A study of discomfort with electrical stimulation. Physical Therapy 1992; 72: $410-421$.

11 Rooney JG, Currier DP, Nitz AJ. Effect of variation in the burst and carrier frequency modes of neuromuscular electrical stimulation on pain perception of healthy subjects. Physical Therapy 1992; 72: $800-807$.

12 Astra Pharma Inc. Instructions for application - Emla Cream. Astra Pharmaceutical Incorporated: Mississauga, Ontario 1995.

13 Rochester L et al. Influence of electrical stimulation of the tibialis anterior muscle in paraplegic subjects. 2. Morphological and histochemical properties. Paraplegia 1995; 33: 514- 522.

14 Braddon RL, Rocco JF. Autonomic dysreflexia: a survey of current treatment. American Journal of Physical Medicine and Rehabilitation 1991; 70: 234-241.

15 Biorad Clinical Division, California 94547. (415-724-7000). Plasma Catecholamines by HPLC Instruction Manual 1991; 956: 136 - 1 (Cat \# 195-6051).

16 Bohannon RW. Manual muscle test scores and dynamometer test scores of knee extension strength. Archives of Physical Medicine and Rehabilitation 1986; 67: 390 .

17 Matthews $\mathbf{J}$ et al. The effects of surface anaesthesia on pain perception during FES. Research Revelations poster presentation, University of Alberta. Edmonton, Canada. March 5, 1994. 